\title{
Prostate Inflammatory Myofibroblastic Tumor
}

National Cancer Institute

\section{Source}

National Cancer Institute. Prostate Inflammatory Myofibroblastic Tumor. NCI

Thesaurus. Code C161042.

A rare inflammatory myofibroblastic tumor that arises from the prostate gland. 